\title{
INFLUÊNCIA DA ADIÇÃO DE DIFERENTES TEORES DE ALUMINATO DE ESTRÔNCIO EM MICROCONCRETOS
}

\author{
Ítalo Ribeiro Gonçalves Lima ${ }^{1 *}$ Yuri Sotero Bomfim Fraga ${ }^{2}$ \\ E-mail : $\underline{\text { italo.engvil@gmail.com * yurisotero.engcivil@gmail.com }}$ \\ ${ }^{1}$ Departamento de engenharia civil, Instituto de ensino superior planalto, Brasília, Brasil \\ ${ }^{2}$ Programa de Pós-graduação em Estruturas e Construção Civil, Universidade de Brasília, Brasília, Brasil
}

\begin{abstract}
RESUMO
Os materiais luminescentes tornaran-se porção restrita do mercado, proporcionando novas oportunidades de pesquisa, geralmente são utilizados para produção de sinalizações de segurança, interruptores, entre outras aplicações. O presente trabalho tem como objetivo analisar o efeito de adições de diferentes teores de aluminato de estrôncio em microconcretos, por meio de dosagens experimentais e estudos teóricos. Foram realizadas quatro incorporações, sendo elas de $25 \%, 50 \%$ e $100 \%$ de aluminato de estrôncio em relação à massa de cimento em argamassa de concreto, com uso de cimento branco em sua composição. A partir disso, foram realizados ensaios de caracterização, flourescência de raio X e luminosidade. Os ensaios indicam que a dosagem de 50\% de aluminato de estrôncio possui melhor desempenho de luminosidade.
\end{abstract}

Palavras chave: Concreto; microconcretos; materiais luminescentes; fluorescência; Aluminato de estrôncio.

\begin{abstract}
Luminescent materials have become a restricted portion of the market, providing new research opportunities, they are generally used for the production of safety signs, switches, among others. The present work aims to analyze the effect of additions of different contents of strontium aluminate in microconcrete, through experimental dosages and theoretical studies, four incorporations of $25 \%, 50 \%$ and $100 \%$ of strontium aluminate were carried out in relation to cement mass in concrete mortar using white cement in its composition, characterization, X-ray flourescence and luminosity tests were carried out. Tests indicate that the dosage of $50 \%$ strontium aluminate has better luminosity performance.
\end{abstract}

Keywords: Concrete; microconcrete; luminescent materials; fluorescence; Strontium Aluminate.

\section{INTRODUÇÃO}

Com o desenvolvimento tecnológico, os materiais fosforescentes, ou também chamados de materiais com resistência prolongada de luminescência, apresentam-se como uma inovação para construção civil. Devido suas inúmeras aplicações, são usados principalmente em tintas 
luminescentes, sinalização de segurança pública, cerâmicas, relógios e brinquedos fosforescentes. Recentemente pesquisadores vem estudando a adição do aluminato de estrôncio em concretos. Segundo Gonzalez (2018), o concreto fotoluminescente tem como principal característica permitir a absorção de energia solar ou artificial durante o dia e reemiti-la à noite.

Pesquisadores, como Rojas-Hernandez et al. (2018), estudaram as diferentes características dos materiais luminescentes, bem como técnicas para aumentar a sua intensidade e duração de brilho. Além disso, Getz et al. (2017) também verificaram as variáveis que afetam as propriedades luminosas desses materiais, tais como calor, contato direto com água e interação com outros materiais.

Em concretos e microconcretos, é estudada adição do aluminato de estrôncio com objetivo de armazenar energia externa e converter em radiação eletromagnética, usualmente na região do UV - Visível-Infravermelho. Esses materiais podem ser excitados por diversas fontes de energias, como por exemplo, a eletromagnética (fotoluminescência), a de radiação ionizante (radioluminescência) e o feixe de elétrons de alta energia. É vasta a aplicação de concretos/argamassas luminescentes, tais como: iluminação de emergência de baixa luminosidade, indicações luminosas, paisagismo decorativo, paver, pavimentações e concretos com fins estruturais.

A Pro-Teq, empresa de origem britânica, desenvolveu uma calçada que emite luz no escuro, sem fontes de energia. O material, denominado Starpath, absorve energia do sol durante o dia e ilumina à noite. O Starpath pode ser usado em qualquer superfície (estradas, passagens de zebra, bordas de piscinas) e possui propriedades de durabilidade importantes, como resistência à chuva e vento, além de ser antiderrapante. Em razão dessa criação, a empresa recebeu o Prêmio Global de Excelência Empresarial de 2013. Trata-se de uma grande invenção que economiza grandes quantias no que diz respeito à iluminação e evita inúmeros acidentes rodoviários (QUIENLOINVENTO, 2014). Para obter transparência e brilho, o grande desafio dos empresários para produzir a calçada de concreto brilhante foi conseguir a transparência, durante o processo de cura, não afetando o produto. Como é bem conhecido, o concreto, ao não permitir que a luz penetre no material, tornase um dos fatores para superar a soluções fotoluminescentes, criando espaços significativos que exigem maior qualidade para utilização final. As novas tecnologias tornaram possíveis o desenvolvimento e produção do presente brilhante especial, otimizando a entrada ao máximo para obter o efeito fotoluminescente, sem alterar propriedades químicas e mecânicas. $\mathrm{O}$ brilho no efeito escuro, ou "brilho noite", perde apenas $10 \%$ de sua potência ao longo de um período de mais de dez anos (RAQUEL OCHOA, 2013).

Por definição, um material luminescente é um material que possui a capacidade de absorver energia e reemiti-la na forma de luz visível. Segundo Callister e Rethwisch (2016), a luminescência possui diferentes classificações dependendo do tempo entre os eventos de absorção e a reemissão, sendo considerado fluorescente o material que consegue reemitir luz visível por menos de 1 segundo, e fosforescente para tempos mais longos. O fenômeno da absorção de energia ocorre quando um elétron passa para um estado de energia chamado excitado, ou seja, de maior energia. Quando esse elétron retorna para um estado de menor energia, ocorre a emissão da luz visível.

Dessa forma, esse artigo tem como objetivo avaliar o efeito da adição de diferentes percentuais de aluminato de estrôncio em microconcretos, analisar o tempo de luminosidade, bem como, definir a relação de dosagem ideal dentre os percentuais analisados.

\section{PROCEDIMIENTO}

\subsection{Materiais}

Para produção dos microconcretos e pastas cimentícias desta pesquisa, foram utilizados os seguintes materiais: Cimento CPB-40 Branco Estrutural - Axton, Aluminato de estrôncio dopado 
com európio e disprósio (SrA12O3) - Luminstant, Areia normal Brasileira - Instituto de pesquisas tecnológicas e Aditivo MC-PowerFlow 1180 - MC-Bauchemie.

\subsection{Métodos}

Foram realizados ensaios de caracterizações dos materiais utilizados para fabricação dos microconcretos em laboratório, conforme resultados presentes na tabela 1.

Tabela 1: Caracterização do cimento Portland da areia normal e do aluminato de estrôncio

\begin{tabular}{|c|c|c|c|c|c|}
\hline Material & \multicolumn{3}{|c|}{ Propriedade } & \multicolumn{2}{|c|}{ Resultados } \\
\hline \multirow{8}{*}{$\begin{array}{c}\text { Cimento CPB-40 } \\
\text { Branco }\end{array}$} & \multirow{2}{*}{\multicolumn{2}{|c|}{ Tempo de pega (min) }} & Início & \multicolumn{2}{|c|}{$1 \mathrm{~h} 40$} \\
\hline & & & Fim & \multicolumn{2}{|c|}{$2 \mathrm{~h} 30$} \\
\hline & \multicolumn{3}{|c|}{ Índice de Brancura (\%) } & \multicolumn{2}{|c|}{$90 \%$} \\
\hline & \multicolumn{3}{|c|}{ Área Específica - Blaine $\left(\mathrm{cm}^{2} / \mathrm{g}\right)$} & \multicolumn{2}{|c|}{4594} \\
\hline & \multicolumn{3}{|c|}{ Massa específica $\left(\mathrm{g} / \mathrm{cm}^{3}\right)$} & \multicolumn{2}{|c|}{3,02} \\
\hline & \multicolumn{3}{|c|}{ Resistência à compressão } & 3 dias & 27,25 \\
\hline & \multirow{2}{*}{\multicolumn{3}{|c|}{$(\mathrm{MPa})$}} & 7 dias & 33,37 \\
\hline & & & & 28 dias & 43,81 \\
\hline \multirow{4}{*}{ Areia Normal } & \multirow{4}{*}{ Fração } & \multirow{4}{*}{ Abertura $(\mathrm{mm})$} & Grossa & \multicolumn{2}{|c|}{2,4 e 1,2} \\
\hline & & & Média grossa & \multicolumn{2}{|c|}{1,2 e 0,6} \\
\hline & & & Média fina & \multicolumn{2}{|c|}{0,6 e 0,3} \\
\hline & & & Fina & \multicolumn{2}{|c|}{0,3 e 0,15} \\
\hline \multirow[t]{2}{*}{ Aluminato de estrôncio } & \multicolumn{3}{|c|}{ Coloração } & \multicolumn{2}{|c|}{$\begin{array}{c}\text { Amarelo } \\
\text { esverdeado }\end{array}$} \\
\hline & \multicolumn{3}{|c|}{ Granulometria } & \multicolumn{2}{|c|}{25 a $35 \mu \mathrm{m}$} \\
\hline
\end{tabular}

O estudo da composição química do aluminato de estrôncio foi realizado através do ensaio de fluorescência de raios-X, de acordo com a análise é possível observar que os elementos $\left(\mathrm{Al}_{2} \mathrm{O}_{3}\right.$ $44,08 \%$ ) e ( $\mathrm{SrO} 50,92 \%$ ) são responsáveis por promover as propriedades fosforescentes nas pastas de cimento deste estudo, exposto na tabela 2.

Tabela 2: Composição química do aluminato de estrôncio - Ensaio de Fluorescência de raio X

\begin{tabular}{|c|c|c|c|c|c|c|c|c|c|c|}
\hline \multirow{2}{*}{\begin{tabular}{|c|c|c|c|c|} 
Material \\
\cline { 2 - 9 }
\end{tabular}} & $\mathrm{Al}_{2} \mathrm{O} 3$ & $\mathrm{CaO}$ & $\mathrm{Rb}_{2} \mathrm{O}$ & $\mathrm{SrO}$ & $\mathrm{Ag}_{2} \mathrm{O}$ & $\mathrm{SnO}_{2}$ & $\mathrm{BaO}^{2}$ & $\mathrm{Eu}_{2} \mathrm{O}_{3}$ & $\mathrm{Dy}_{2} \mathrm{O}_{3}$ & $\mathrm{CO}_{2}$ \\
\hline Aluminato de estrôncio & 44,08 & 0,854 & 0,081 & 50,92 & 0,179 & 0,017 & 0,337 & 0,597 & 0,775 & 2,1 \\
\hline
\end{tabular}

Para confecção das argamassas foram observados os parâmetros da NBR7215:2019, os corpos de prova são dimensionados para uma parte de cimento e três partes de areia normalizada, com relação água/cimento de 0,48. Para realização do teste comparativo foram dosados quatro traços de argamassa variando a quantidade de aluminato de estrôncio em relação a massa de cimento, sendo uma argamassa referência, somente com utilização do cimento Portland - Branco e para os demais traços foram adicionados em 25\%,50\% e 100\% do aluminato de estrôncio, conforme tabela 3 . 
Tabela 3: Microconcretos

\begin{tabular}{|c|c|c|c|c|c|c|c|}
\hline \multicolumn{2}{|c|}{ Microconcreto } & \multicolumn{4}{|c|}{ Areia normal (g) } & \multirow{2}{*}{$\begin{array}{c}\text { Água } \\
\text { (g) }\end{array}$} & \multirow{2}{*}{$\begin{array}{l}\text { (aluminato de } \\
\text { estrôncio) (g) }\end{array}$} \\
\hline $\begin{array}{c}\text { Dosage } \\
\mathrm{m}\end{array}$ & $\begin{array}{c}\text { CP-Branco } \\
\text { (g) }\end{array}$ & Fina & $\begin{array}{c}\text { Média } \\
\text { fina }\end{array}$ & $\begin{array}{l}\text { Média } \\
\text { grossa }\end{array}$ & Grossa & & \\
\hline REF & 624 & 468 & 468 & 468 & 468 & 300 & - \\
\hline AE 25 & 624 & 468 & 468 & 468 & 468 & 300 & 156 \\
\hline AE 50 & 624 & 468 & 468 & 468 & 468 & 300 & 312 \\
\hline AE 100 & 624 & 468 & 468 & 468 & 468 & 300 & 624 \\
\hline
\end{tabular}

Para análise de luminosidade dos microconcretos, foram moldados corpos de prova conforme parâmetros da NBR 7215 (ABNT, 2019). Para tal fim, a mistura dos materiais foi realizada em um misturador planetário com capacidade de $5 \mathrm{~L}$ por $1 \mathrm{~min}$ na velocidade baixa (140 rpm) e depois 30 segundos na velocidade alta $(285 \mathrm{rpm})$, após este procedimento a mistura ficou em repouso durante 1 minuto e logo em seguida misturada novamente em velocidade alta por mais 1 minuto. Os corpos de prova foram moldados conforme parâmetro da NBR 7215 (ABNT, 2019), 4 camadas sendo distribuídos 30 golpes em cada. Para cada dosagem foram moldados 7 corpos de prova, nos quais foram utilizados moldes cilíndricos com $50 \mathrm{~mm}$ de diâmetro x $100 \mathrm{~mm}$. Os corpos de prova foram armazenados em cura submersa em água saturada de cal.

\subsection{Pastas de cimento}

A preparação das pastas seguiu as recomendações da NBR 16606 (ABNT, 2017), com algumas adaptações. Em função da quantidade de aluminato de estrôncio disponível para confecção das pastas, foi reduzido o quantitativo de material estabelecido na norma, contudo, foi preservado a relação água/aglomerante. Foram realizadas as confecções de quatro pastas, sendo uma referência, e nas outras adicionados os teores de $25 \%, 50 \%$ e $100 \%$ de aluminato de estrôncio. Para manter a consistência da mistura, foi adicionado aditivo superplastificante. Em relação à pasta de referência e a pasta $\mathrm{AE} 25$, foi adicionado $0,001 \%$ de aditivo em relação a massa de cimento, para as pastas AE50 e AE100 foram adicionados 0,003\%.

Em uma cuba de aço inox foram adicionados todos os materiais líquidos (água e aditivo superplastificante) e posteriormente foi inserido o Cimento Portland Branco. Após o contato do cimento com a mistura, foram contabilizados 30 segundos em repouso. Depois foi realizada a mistura com o auxílio do misturador planetário durante 30 segundos na velocidade lenta. $\mathrm{O}$ misturador foi desligado por 60 segundos, sendo realizada a raspagem das paredes internas da cuba durante os primeiros 30 segundos. Por fim, o misturador planetário foi ligado na velocidade alta por 60 segundos. A partir do desenvolvimento dessas pastas, foram moldados quatro corpos de prova. Após a moldagem, os corpos de prova foram colocados em câmara úmida por 24 horas e depois em cura submersa em água saturada com cal até a idade de ensaio.

Após os 28 dias de cura foi realizada a fragmentação das pastas com objetivo de paralisar a hidratação das pastas, utilizando uma das metodologias de Scrivener; Snellings; Lothenbach (2016). O procedimento consistiu na imersão dos fragmentos em isopropanol por 6 horas e secagem em estufa à $40 \pm 1^{\circ} \mathrm{C}$ por 24 horas. Posteriormente, as amostras foram armazenadas em um recipiente contendo sílica gel, para evitar a hidratação, e cal sodada, para evitar a carbonatação, até o dia de realização dos ensaios microestruturais. Esses fragmentos das pastas foram moídos com auxílio do almofariz de ágata, e peneirados na peneira de abertura de $0,075 \mathrm{~mm}$. Estes foram 
utilizados posteriormente para os ensaios de fluorescência de raios X (FRX). O processo de paralisação da hidratação das pastas é mostrado na figura 1.
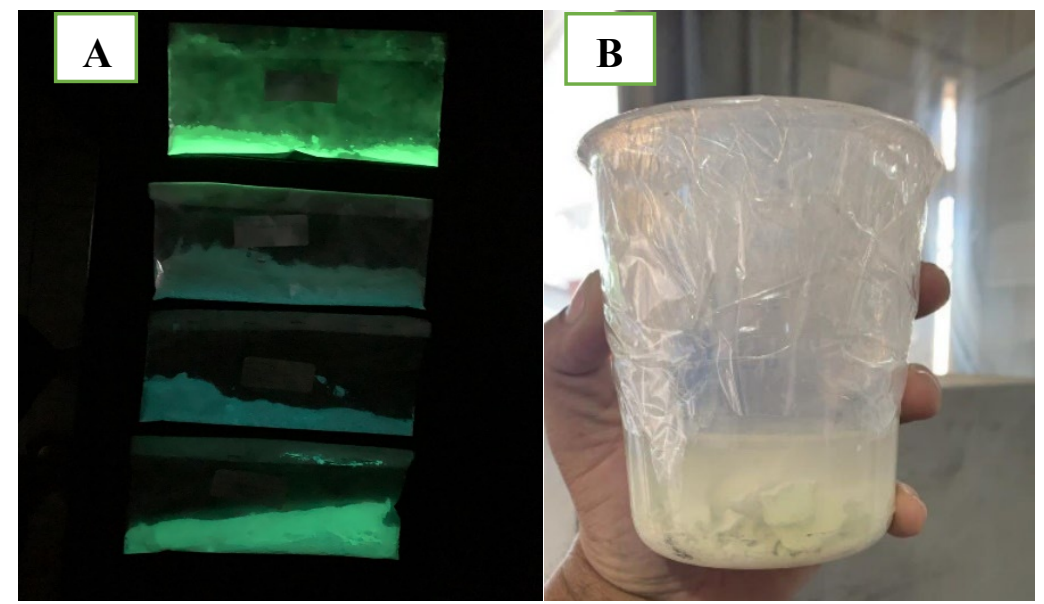

Figura 1: Pastas de cimento moídas (a), processo de paralisação da hidratação (b)

Não foram realizadas medições de luminosidade nas pastas de cimentos com adição do aluminato de estrôncio, contudo, as pastas foram expostas durante 30 minutos a uma lâmpada fluorescente ultravioleta. Conforme figura 1, é possível observar o fenômeno de absorção de energia ocorre quando um elétron passa para um estado de energia chamado excitado, ou seja, de maior energia. Quando esse elétron retorna para um estado de menor energia, ocorre a emissão da luz visível.

Na tabela 4 é possível verificar a composição química das pastas de cimento, realizadas através do ensaio de fluorescência de raio X (FRX), bem como a presença do aluminato de estrôncio.

Tabela 4: Fluorescência de raio $\mathrm{X}$ das pastas

\begin{tabular}{|c|c|c|c|c|c|c|c|c|c|c|c|c|c|c|}
\hline Pastas & $\begin{array}{c}\% \\
\mathrm{Al}_{2} \mathrm{O} \\
3\end{array}$ & $\begin{array}{c}\% \\
\mathrm{Ca} \\
\mathrm{O}\end{array}$ & $\begin{array}{c}\% \\
\mathrm{Fe}_{2} \\
\mathrm{O}_{3}\end{array}$ & $\begin{array}{c}\% \\
K_{2} \\
\mathbf{O}\end{array}$ & $\begin{array}{c}\% \\
\mathrm{Na}_{2} \\
\mathrm{O}\end{array}$ & $\begin{array}{c}\% \\
\mathrm{Mg} \\
\mathrm{O}\end{array}$ & $\begin{array}{c}\% \\
\mathrm{SiO} \\
2\end{array}$ & $\begin{array}{c}\% \\
\text { SO } \\
3\end{array}$ & $\begin{array}{c}\% \\
P_{2} \mathrm{O} \\
5\end{array}$ & $\begin{array}{l}\% \\
\mathrm{Cl}\end{array}$ & $\begin{array}{c}\% \\
\mathrm{TiO} \\
2 \\
\end{array}$ & $\begin{array}{c}\% \\
\mathrm{NiO}\end{array}$ & $\begin{array}{c}\% \\
\text { SrO }\end{array}$ & $\begin{array}{c}\% \\
\text { Fluor }\end{array}$ \\
\hline REF & 2,51 & 75,6 & 0,34 & 0,15 & 0,11 & 1,47 & 16,1 & $\begin{array}{c}2,7 \\
8\end{array}$ & 0,07 & $\begin{array}{c}0,0 \\
4\end{array}$ & 0,19 & 0,02 & 0,15 & 0,53 \\
\hline AE 25 & 12,5 & 58,5 & 0,22 & 0,16 & 0,08 & 1,02 & 11,5 & $\begin{array}{c}2,2 \\
9 \\
\end{array}$ & 0,07 & $\begin{array}{c}0,0 \\
4 \\
\end{array}$ & 0,1 & 0 & 13,4 & 0 \\
\hline AE 50 & 20,2 & 47,4 & 0,19 & 0,05 & 0 & 1,05 & 7,63 & \begin{tabular}{|c|}
2,1 \\
1 \\
\end{tabular} & 0,12 & $\begin{array}{c}0,0 \\
3 \\
\end{array}$ & 0,08 & 0,04 & 20,9 & 0 \\
\hline AE 100 & 26,2 & 35,4 & 0,17 & 0,08 & 0 & 0,79 & 6,23 & $\begin{array}{c}1,5 \\
5\end{array}$ & 0,13 & $\begin{array}{c}0,0 \\
4\end{array}$ & 0,07 & 0 & 29,1 & 0 \\
\hline
\end{tabular}

Em função dos dados analisados na tabela 4 destaca-se a presença do aluminato de estrôncio nas pastas AE25, AE50 e AE100 em relação à pasta REF, o que evidencia que o fenômeno de fosforescência se resulta através da presença destes componentes químicos que foram adicionadas às pastas.

Segundo $\mathrm{Hu}$ et. al (2018), a luminescência intensa dos aluminatos de estrôncio se deve pela ocorrência de uma transição dos íons $\mathrm{Eu}^{+2}$ de $4 \mathrm{f}^{6} 5 \mathrm{~d}$ para $4 \mathrm{f}^{7}$. Para realização da análise de luminosidade dos corpos de prova, foi utilizada uma lâmpada fluorescente ultravioleta, onde os corpos de prova foram expostos a uma dose de excitação de $8 \mathrm{~h}$. Após o tempo transcorrido foram observados o tempo de remissão de luz visível. 


\section{RESULTADOS}

\section{Análise de luminosidade}

Foi observado que nos primeiros 30 minutos após a excitação de 8 horas de exposição à lâmpada fluorescente ultravioleta, não houve diminuição da intensidade de luminosidade que os corpos de prova reemitiam. Após os 30 minutos a dosagem de AE25 apresentou decaimento mais abrupto dentre os materiais, cessando a reemissão de luminosidade visível logo após uma hora e trinta minutos. Para as dosagens de AE50 e AE100, foi observado que somente depois de três horas de reemissão de luminosidade o material começou apresentar vagaroso decaimento cessando sua luminosidade quatro horas e trinta minutos após a retirada da fonte de excitação, conforme tabela 5 .

Tabela 5: Tempo de exposição lâmpada fluorescente ultravioleta

\begin{tabular}{|c|c|c|}
\hline Dosagem & Tempo de exposição & Tempo de reemissão de luz \\
\hline AE 25 & $8 \mathrm{~h}$ & $1 \mathrm{~h} 30$ \\
\hline AE 50 & $8 \mathrm{~h}$ & $4 \mathrm{~h} 30$ \\
\hline AE 100 & $8 \mathrm{~h}$ & $4 \mathrm{~h} 30$ \\
\hline
\end{tabular}

De acordo com Lisensky, Patel e Reich (1996), os materiais luminescentes podem apresentar diferentes comportamentos de decaimento luminoso. Conforme a tabela 5 distinguisse a reemissão de luminosidade entre adição de menores teores de aluminato de estrôncio e maiores adições. Conforme gráfico apresentado na figura 2 é possível observar o tempo de reemissão e decaimento de cada material.



Figura 2: Gráfico de tempo de reemissão de luminosidade

Zhao, Li e Wang (2013) testaram a adição de 3 pós luminescentes distintos diretamente à mistura de cimento, água, areia e brita, variando a concentração de 2, 4, 6 e $8 \%$ da massa de cimento em gramas de material luminescente. Os resultados obtidos demonstram que a fosforescência foi possível e obteve decaimento exponencial. Os materiais apresentaram diferentes comportamentos de luminosidade, em concordância com gráfico apresentado na figura 2. Destaca-se que ao cessar 
a fonte de excitação nos 30 minutos iniciais, os microconcretos apresentaram pico máximo de luminosidade visível conforme figura 3.

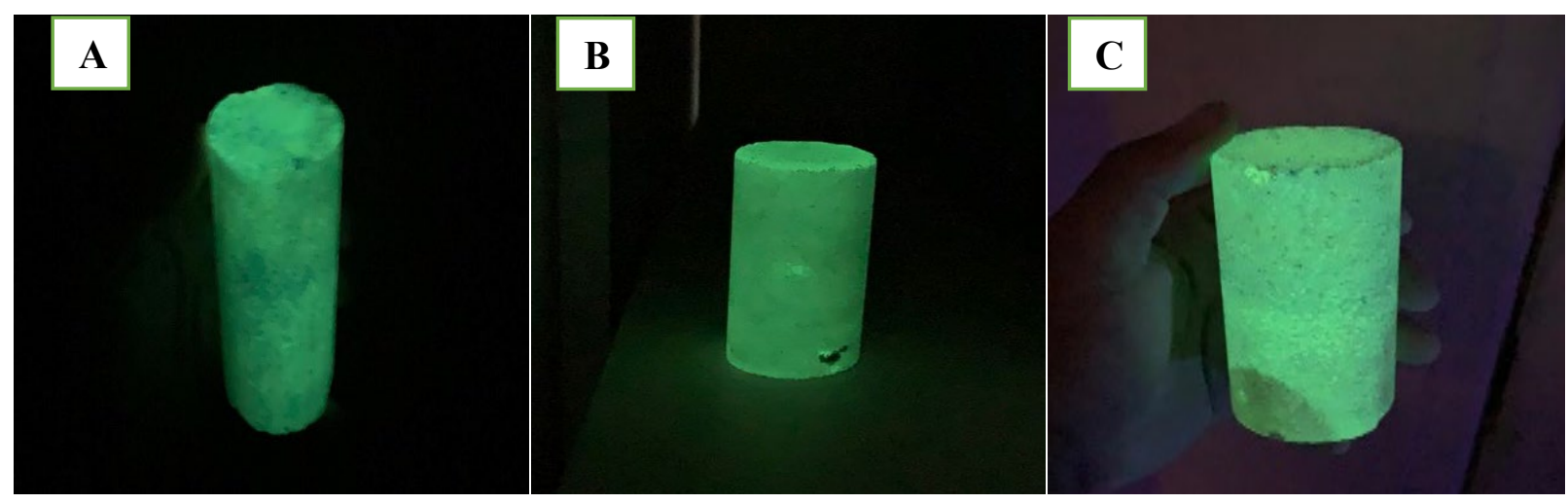

Figura 3: Microconcretos (a) AE25, (b) AE50 e (c) AE100 pico máximo de reemissão de luminosidade

É possível observar, conforme figura 3, que os microconcretos não apresentaram diferença de intensidade luminosidade visível em até 30 minutos. Na figura 4 é possível observar o decaimento de luminosidade.



Figura 4: Microconcretos (a) AE25, (b) AE50 e (c) AE100 decaimento de reemissão de luminosidade

Após 30 minutos de reemissão de luz os microconcretos apresentaram diferentes intensidade de brilho e tempo de reemissão. A AE25 apresentou abrupto decaimento em relação a AE50 e AE100, apresentando tempo de reemissão de $1 \mathrm{~h} 30 \mathrm{~min}$. Dentre todos as proporções testadas, o aluminato de estrôncio de AE50 e AE100 apresentaram o mesmo desempenho nos aspectos de tempo de luminosidade, portanto, a quantidade de $\left(\mathrm{Al}_{2} \mathrm{O}_{3}\right)$ 20,20\% e (SrO) 20,93\% presente na pasta de cimento e observado pelo ensaio de fluorescência de raio $\mathrm{X}$ (FRX), se enquadra como a dosagem com melhor performance. 


\section{CONCLUSÃO}

Em função do ensaio de flourescência de raio X (FRX), foi possível identificar a composição química das pastas dos microconcretos, evidenciando que o $\left(\mathrm{SrAl}_{2} \mathrm{O}_{3}\right)$ e $(\mathrm{SrO})$ são os elemetos responsáveis por produzir a propiedade de fosforecencia nas pastas e microconcretos. Também é possível afirmar, a partir dos estudos de luminosidade realizados, que a dosagem de AE50 de aluminato de estrôncio obteve melhor performasse de luminosidade em realação às demais dosagens, uma vez que a dosagem de AE25 obteve desempenho de 1h30min de reemissão de luminosidade, e para as dosagens de AE50 e AE100, o desempenho foi de 4h30min de reemissão de luminosidade.

Dessa forma, é possível inferir que o aluminato de estrôncio possui influência direta no desempenho luminoso de compostos cimentícios.

\section{AGRADECIMENTOS}

Os autores agradecem as empresas Supermix Concreto S/a e Votorantim Cimentos S/a pelo fornecimento do laboratório para realização de ensaios de caracterização das pastas e microconcretos deste estudo.

\section{REFERÊNCIAS}

Associação Brasileira de Normas Técnicas. (2016). NBR13276: Argamassa para assentamento de paredes e revestimento de paredes e teto - Determinação do índice de consistência. Rio de Janeiro.

Associação Brasileira de Normas Técnicas. (2005). NBR13279: Argamassa para assentamento de paredes e revestimento de paredes e teto - Determinação da resistência à compressão. Rio de Janeiro.

Associação Brasileira de Normas Técnicas. (2016). NBR16541: Argamassa para assentamento de paredes e revestimento de paredes e teto - Preparo da mistura para realização de ensaios. Rio de Janeiro.

Associação Brasileira de Normas Técnicas. (2019). NBR7215: Cimento Portland-Determinação da resistência à compressão. Rio de Janeiro.

Associação Brasileira de Normas Técnicas. (2018) NBR 16697: cimento Portland: requisitos. Rio de Janeiro.

Associação Brasileira de Normas Técnicas. (1993) NBR NM. 12989: Cimento Portland branco. Rio de Janeiro.

Braschemical. (2019), “Aluminato de Estrôncio dopado com Európio” Literatura Técnica. São Paulo.

Callister Jr., W. D.; rethwisch, d. g. (2016) “Ciência e engenharia dos materiais. LTC, $9^{\circ}$ ed". p.912 
Getz, William A. et al. (2017) Erasing the glow in the dark: controlling the trap and release of electrons in phosphorescent materials. Journal of Chemical Education, [s.1.], v. 95, n. 2, p.295-299 https://pubs.acs.org/doi/10.1021/acs.jchemed.7b00744_Acesso em: 18 maio. 2021

Gonzalez, J.a.m.; J.1.m. (2018) "Influência de pedras fotoluminescentes em iluminação e propriedades mecânica do concreto". Trujillo, Peru.

HU, Xiaowu et al. (2018) "Preparation and properties of Eu and Dy co-doped strontium aluminate long afterglow nanomaterials. Ceramics International" Ceramics International, [s.1.], v. 44, n. 7, p.7535-7544 Disponível em: https://doi.org/10.1016/j.ceramint.2018.01.157 Acesso em: 29 maio. 2021

Kirchheim, A. P. (2003) "Concreto de cimento Portland branco estrutural: avaliação de carbonatação e absorção capilar. Dissertação" (Mestrado em Engenharia) - Universidade Federal do Rio Grande do Sul.

Lisensky, George C.; Patel, Manish N.; Reich, Megan L. "Experiments with glowin-the-dark toys: kinetics of doped zns phosphorescence” Journal of Chemical

Moreira, D. Cimento que emite luz. FNE Federação nacional dos engenheiros. Disponível em https://www.fne.org.br/index.php/comunicacao/jornal-fne/jornal-edicao-191/4761-cimento-que-

emite-luz Acesso em: 29 maio. 2021.

Piovesan, A.Z. (2009) "Estudo sobre a influência da adição de pigmentos em propriedades de durabilidade e na cromacidade do concreto de cimento Portland branco" Dissertação para obtenção do título de mestre em engenharia civil. Programa de pós-graduação do Rio grande do Sul. Porto Alegre.

Quienloinvento. (2014) "Starpath, Pavimento Luminescente". Disponível em: https://quienloinvento.wordpress.com/2014/10/18/pavimento-luminiscente/ Acesso em: 12 maio 2021.

Raquel Ochoa. (2013) “Um mundo brilhante debaixo de seus pés”. Disponível em: < http://www.clubedoconcreto.com.br/2013/10/um-mundo-brilhante-debaixo-de-seus-pes.html> Acesso em: 12 maio 2021.

Scrivener, K.; Snellings, R.; Lothenbach, B. (2016) A practical guide to microstructural analysis of cementitious materials. CRC PRESS.

Smith, W. F., Hashemi, J.(2012) "Fundamentos de engenharia e ciência dos materiais. AMGH, $5^{a}$ ed". 